Article

\title{
Schrödinger Theory of Electrons in Electromagnetic Fields: New Perspectives
}

\author{
Viraht Sahni ${ }^{1, *}$ and Xiao-Yin Pan ${ }^{2}$ \\ 1 The Graduate School of the City University of New York, New York, NY 10016, USA \\ 2 Department of Physics, Ningbo University, Ningbo 315211, China; panxiaoyin@nbu.edu.cn \\ * Correspondence: vsahni@brooklyn.cuny.edu; Tel.: +1-718-951-5000 (ext. 2866)
}

Academic Editor: Jianmin Tao

Received: 20 January 2017; Accepted: 1 March 2017; Published: 9 March 2017

\begin{abstract}
The Schrödinger theory of electrons in an external electromagnetic field is described from the new perspective of the individual electron. The perspective is arrived at via the time-dependent "Quantal Newtonian" law (or differential virial theorem). (The time-independent law, a special case, provides a similar description of stationary-state theory). These laws are in terms of "classical" fields whose sources are quantal expectations of Hermitian operators taken with respect to the wave function. The laws reveal the following physics: (a) in addition to the external field, each electron experiences an internal field whose components are representative of a specific property of the system such as the correlations due to the Pauli exclusion principle and Coulomb repulsion, the electron density, kinetic effects, and an internal magnetic field component. The response of the electron is described by the current density field; (b) the scalar potential energy of an electron is the work done in a conservative field. It is thus path-independent. The conservative field is the sum of the internal and Lorentz fields. Hence, the potential is inherently related to the properties of the system, and its constituent property-related components known. As the sources of the fields are functionals of the wave function, so are the respective fields, and, therefore, the scalar potential is a known functional of the wave function; (c) as such, the system Hamiltonian is a known functional of the wave function. This reveals the intrinsic self-consistent nature of the Schrödinger equation, thereby providing a path for the determination of the exact wave functions and energies of the system; (d) with the Schrödinger equation written in self-consistent form, the Hamiltonian now admits via the Lorentz field a new term that explicitly involves the external magnetic field. The new understandings are explicated for the stationary state case by application to two quantum dots in a magnetostatic field, one in a ground state and the other in an excited state. For the time-dependent case, the evolution of the same states of the quantum dots in both a magnetostatic and a time-dependent electric field is described. In each case, the satisfaction of the corresponding "Quantal Newtonian" law is demonstrated.
\end{abstract}

Keywords: Schrödinger equation for electrons; self-consistency; “Quantal Newtonian” laws

\section{Introduction}

In this paper, we explain new understandings [1] of Schrödinger theory of the electronic structure of matter, and of the interaction of matter with external static and time-dependent electromagnetic fields. Matter-atoms, molecules, solids, quantum wells, two-dimensional electron systems such as at semiconductor heterojunctions, quantum dots, etc.-is defined here as a system of $N$ electrons in an external electrostatic field $\mathcal{E}(\mathbf{r})=-\nabla v(\mathbf{r})$, where $v(\mathbf{r})$ is the scalar potential energy of an electron. The added presence of a magnetostatic field $\mathcal{B}(\mathbf{r})=\boldsymbol{\nabla} \times \mathbf{A}(\mathbf{r})$, with $\mathbf{A}(\mathbf{r})$ the vector potential, corresponds to the Zeeman, Hall, Quantum Hall, and magneto-caloric effects, magnetoresistance, etc. The interaction of radiation with matter such as laser-atom interactions, photo-electric effects at metal surfaces, etc., are described by the case of external time-dependent electromagnetic fields. 
The insights are arrived at by describing Schrödinger theory from the perspective [2,3] of the individual electron. This perspective is arrived at via the "Quantal Newtonian" second law [4-7] (or the time-dependent differential virial theorem) for each electron, with the first law [8-10] being a description of stationary-state theory. The laws are a description of the system [2,3] in terms of "classical" fields whose sources are quantal in that they are expectations of Hermitian operators taken with respect to the wave function (For the origin of these ideas see [11-13]). This manner of depiction makes the description of Schrödinger theory tangible in the classical sense. The new understandings described are a consequence of these "Quantal Newtonian" laws.

A principal insight into Schrödinger theory arrived at is that the Schrödinger equation can be written in self-consistent form. To explain what we mean, consider first the stationary-state case. It is proved via the "Quantal Newtonian" first law, that for arbitrary state, the Hamiltonian $\hat{H}$ for the system of electrons in a static electromagnetic field is a functional of the wave function $\Psi$, i.e., $\hat{H}=\hat{H}[\Psi]$. Hence, the corresponding Schrödinger equation can be written as $\hat{H}[\Psi] \Psi=E \Psi$. Thus, the eigenfunctions $\Psi$ and eigenenergies $E$ of the Schrödinger equation can be obtained self-consistently. This form of eigenvalue equation is mathematically akin to that of Hartree-Fock and Hartree theories in which the corresponding Hamiltonian $\hat{H}^{\mathrm{HF}}$ is a functional of the single particle orbitals $\phi_{i}$ of the Slater determinant wave function. The corresponding integro-differential eigenvalue equations are then $\hat{H}^{\mathrm{HF}}\left[\phi_{i}\right] \phi_{i}=\epsilon_{i} \phi_{i}$. The orbitals $\phi_{i}$ and the eigenenergies $\epsilon_{i}$ are obtained by self-consistent solution of the equation $[14,15]$. There are other formalisms, as for example within the context of local effective potential theory where the electrons are replaced by noninteracting fermions, for which the solution is also obtained self-consistently. Such theories are Kohn-Sham density functional theory [16,17], the Optimized Potential Method [18,19], Quantal density functional theory (QDFT) [2,3], and the Hartree and Pauli-correlated approximations within QDFT [3,20,21]. In general, eigenvalue equations of the form $\hat{L}[\zeta] \zeta=\lambda \zeta$, where $\hat{L}$ is an integro-differential operator, are solved in an iterative self-consistent manner. However, whereas in Hartree-Fock and local effective potential theories, the self-consistency is for the single particle orbitals $\phi_{i}$ leading to the Slater determinant wave function, the self-consistent solution of the Schrödinger equation leads to the many-electron fully-interacting system wave function $\Psi$ and energy $E$. In the time-dependent case, it is shown via the "Quantal Newtonian" second law that the Hamiltonian $\hat{H}(t)=\hat{H}[\Psi(t)]$, so that the self-consistent form of the Schrödinger equation is $\hat{H}[\Psi(t)] \Psi(t)=i \partial \Psi(t) / \partial t$. Thus, in this instance, it is the evolution of the many-electron time-dependent wave function $\Psi(t)$ that is obtained self-consistently.

Other understandings achieved show that the scalar potential energy of an electron $v(\mathbf{r})$ is the work done in a conservative field $\mathcal{F}(\mathbf{r})$. The components of this field are separately representative of properties of the system such as the correlations due to the Pauli exclusion principle and Coulomb repulsion, the electron density, kinetic effects, an internal magnetic field contribution, and the Lorentz field. The constituent property-related components of the potential $v(\mathbf{r})$ are thus known. The components of the field $\mathcal{F}(\mathbf{r})$ are each derived from quantal sources that are expectation values of Hermitian operators taken with respect to the wave function $\Psi$. Thus, the potential $v(\mathbf{r})$, (and hence the Hamiltonian), is a known functional of the wave function. Finally, the presence of the Lorentz field in the expression for $v(\mathbf{r})$, admits a term involving the magnetic field $\mathcal{B}(\mathbf{r})$ in the Schrödinger equation as written in self-consistent form. These insights all lead to a fundamentally different way of thinking of the Schrödinger equation.

The new physics is explicated for the stationary-state case by application to two quantum dots or two-dimensional "artificial atoms" in a magnetostatic field with one being in a ground state and the other in a first excited singlet state. For the time-dependent case, the same states of these quantum dots in a magnetostatic field perturbed by a time-dependent electric field are considered. In each case, the corresponding "Quantal Newtonian" law is shown to be satisfied.

We begin with a brief summary of the manner in which Schrödinger theory is presently understood and practiced. For this, consider stationary-state theory for a system of $N$ electrons in an external electrostatic field $\mathcal{E}(\mathbf{r})=-\boldsymbol{\nabla} v(\mathbf{r})$ and magnetostatic field $\mathcal{B}(\mathbf{r})=\boldsymbol{\nabla} \times \mathbf{A}(\mathbf{r})$. 
The Schrödinger equation in atomic units (charge of electron $-e,|e|=\hbar=m=1$ ) together with the assumption of $c=1$ is

$$
\left[\frac{1}{2} \sum_{i}\left(\hat{\mathbf{p}}_{i}+\mathbf{A}\left(\mathbf{r}_{i}\right)\right)^{2}+\frac{1}{2} \sum_{i, j}^{\prime} \frac{1}{\left|\mathbf{r}_{i}-\mathbf{r}_{j}\right|}+\sum_{i} v\left(\mathbf{r}_{i}\right)\right] \Psi(\mathbf{X})=E \Psi(\mathbf{X})
$$

where the terms of the Hamiltonian are the physical kinetic, electron-interaction potential, and scalar potential energy operators; $\{\Psi(\mathbf{X}) ; E\}$ the eigenfunctions and eigenvalues; $\mathbf{X}=\mathbf{x}_{1}, \mathbf{x}_{2}, \ldots, \mathbf{x}_{N} ; \mathbf{x}=\mathbf{r} \sigma$; and $(\mathbf{r} \sigma)$ the spatial and spin coordinates.

We note the following salient features of the above Schrödinger equation:

(a) as a consequence of the correspondence principle, it is the vector potential $\mathbf{A}(\mathbf{r})$ and not the magnetic field $\mathcal{B}(\mathbf{r})$ that appears in it. This fact is significant, and is expressly employed to explain, for example, the Bohm-Aharonov [22] effect in which a vector potential can exist in a region of no magnetic field. The magnetic field $\mathcal{B}(\mathbf{r})$ appears in the Schrödinger equation only following the choice of gauge;

(b) the characteristics of the potential energy operator $v(\mathbf{r})$ are the following:

(i) for the $\mathrm{N}$-electron system, it is assumed that the canonical kinetic and electron-interaction potential energy operators are known. As such, the potential $v(\mathbf{r})$ is considered an extrinsic input to the Hamiltonian.

(ii) the potential energy function $v(\mathbf{r})$ is assumed known, e.g., it could be Coulombic, harmonic, Yukawa, etc.

(iii) by assumption, the potential $v(\mathbf{r})$ is path-independent.

With the Hamiltonian operator known, the Schrödinger differential equation is then solved for $\{\Psi(\mathbf{X}) ; E\}$. Physical observables are determined as expectations of Hermitian operators taken with respect to $\Psi(\mathbf{X})$.

We initially focus on the stationary-state case. In Section 2, we briefly describe the single-electron perspective of time-independent Schrödinger theory via the "Quantal Newtonian" first law. The explanation of the new understandings achieved is given in Section 3. These ideas are further elucidated in Section 4 by the example of a quantum dot in a magnetostatic field. Both a quantum dot in a ground state and one in an excited state are considered. The extension to the time-dependent case via the "Quantal Newtonian" second law with examples is discussed in Section 5. Concluding remarks are made in Section 6 together with a comparison of the self-consistent method and the variational [23] and constrained-search variational methods [24-26] for the determination of the wave function.

\section{Stationary State Theory: "Quantal Newtonian" First Law}

In order to better understand the "Quantal Newtonian" laws for each electron, we first draw a parallel to Newton's laws for the individual particle. Hence, consider a system of $N$ classical particles that obey Newton's third law, exert forces on each other that are equal and opposite, directed along the line joining them, and are subject to an external force. Then, Newton's second law for the $i$ th particle is

$$
\mathbf{F}_{i}^{\mathrm{ext}}+\sum_{j}^{\prime} \mathbf{F}_{j i}=d \mathbf{p}_{i} / d t
$$

where $\mathbf{F}_{i}^{\text {ext }}$ is the external force, $\mathbf{F}_{j i}$ the internal force on the $i$ th particle due to the $j$ th particle, and $\mathbf{p}_{i}$ the linear momentum response of the $i$ th particle to these forces. In summing Equation (2) over all the particles, the internal force contribution vanishes, leading to Newton's second law.

Newton's first law for the $i$ th particle is

$$
\mathbf{F}_{i}^{\text {ext }}+\sum_{j}^{\prime} \mathbf{F}_{j i}=0 .
$$


Again, on summing over all the particles, the internal force component vanishes leading to Newton's first law.

The "Quantal Newtonian" first law for the quantum system described by Equation (1) - (the counterpart to Newton's first law for each particle)—states that the sum of the external $\mathcal{F}^{\text {ext }}(\mathbf{r})$ and internal $\mathcal{F}^{\text {int }}(\mathbf{r})$ fields experienced by each electron vanish $[2,27,28]$ :

$$
\mathcal{F}^{\text {ext }}(\mathbf{r})+\mathcal{F}^{\text {int }}(\mathbf{r})=0
$$

Just as the Schrödinger equation Equation (1), so is the "Quantal Newtonian" first law valid for arbitrary state. It is gauge invariant and derived employing the continuity condition $\boldsymbol{\nabla} \cdot \mathbf{j}(\mathbf{r})=0$. Here, $\mathbf{j}(\mathbf{r})$ is the physical current density which is the expectation

$$
\mathbf{j}(\mathbf{r})=\langle\Psi(\mathbf{X})|\hat{\mathbf{j}}(\mathbf{r})| \Psi(\mathbf{X})\rangle,
$$

with the current density operator

$$
\hat{\mathbf{j}}(\mathbf{r})=\frac{1}{2 i} \sum_{k}\left[\boldsymbol{\nabla}_{\mathbf{r}_{k}} \delta\left(\mathbf{r}_{k}-\mathbf{r}\right)+\delta\left(\mathbf{r}_{k}-\mathbf{r}\right) \boldsymbol{\nabla}_{\mathbf{r}_{k}}\right]+\hat{\rho}(\mathbf{r}) \mathbf{A}(\mathbf{r}),
$$

and the density operator

$$
\hat{\rho}(\mathbf{r})=\sum_{k} \delta\left(\mathbf{r}_{k}-\mathbf{r}\right)
$$

The external field is the sum of the electrostatic $\mathcal{E}(\mathbf{r})$ and Lorentz $\mathcal{L}(\mathbf{r})$ fields [2,27]:

$$
\mathcal{F}^{\mathrm{ext}}(\mathbf{r})=\mathcal{E}(\mathbf{r})-\mathcal{L}(\mathbf{r})=-\nabla v(\mathbf{r})-\mathcal{L}(\mathbf{r}),
$$

where $\mathcal{L}(\mathbf{r})$ is defined in terms of the Lorentz "force" $\ell(\mathbf{r})$ and density $\rho(\mathbf{r})$ as

$$
\mathcal{L}(\mathbf{r})=\frac{\ell(\mathbf{r})}{\rho(\mathbf{r})}
$$

where

$$
\ell(\mathbf{r})=\mathbf{j}(\mathbf{r}) \times \mathcal{B}(\mathbf{r})
$$

and the density is the expectation

$$
\rho(\mathbf{r})=\langle\Psi(\mathbf{X})|\hat{\rho}(\mathbf{r})| \Psi(\mathbf{X})\rangle .
$$

The internal field $\mathcal{F}^{\text {int }}(\mathbf{r})$ is the sum of the electron-interaction $\mathcal{E}_{\text {ee }}(\mathbf{r})$, kinetic $\mathcal{Z}(\mathbf{r})$, differential density $\mathcal{D}(\mathbf{r})$, and internal magnetic $\mathcal{I}(\mathbf{r})$ fields [2,27]:

$$
\mathcal{F}^{\text {int }}(\mathbf{r})=\mathcal{E}_{\mathrm{ee}}(\mathbf{r})-\mathcal{Z}(\mathbf{r})-\mathcal{D}(\mathbf{r})-\mathcal{I}(\mathbf{r})
$$

These fields are defined in terms of the corresponding "forces" $\mathbf{e}_{\mathrm{ee}}(\mathbf{r}), \mathbf{z}(\mathbf{r}), \mathbf{d}(\mathbf{r})$, and $\mathbf{i}(\mathbf{r})$. (Each "force" divided by the (charge) density $\rho(\mathbf{r})$ constitutes the corresponding field). Thus,

$$
\mathcal{E}_{\mathrm{ee}}(\mathbf{r})=\frac{\mathbf{e}_{\mathrm{ee}}(\mathbf{r})}{\rho(\mathbf{r})} ; \mathcal{Z}(\mathbf{r})=\frac{\mathbf{z}(\mathbf{r})}{\rho(\mathbf{r})} ; \mathcal{D}(\mathbf{r})=\frac{\mathbf{d}(\mathbf{r})}{\rho(\mathbf{r})} ; \mathcal{I}(\mathbf{r})=\frac{\mathbf{i}(\mathbf{r})}{\rho(\mathbf{r})}
$$

The "force" $\mathbf{e}_{\mathrm{ee}}(\mathbf{r})$, representative of electron correlations due to the Pauli exclusion principle and Coulomb repulsion, is obtained via Coulomb's law via its quantal source, the pair-correlation function $P\left(\mathbf{r r}^{\prime}\right)$ :

$$
\mathbf{e}_{\mathrm{ee}}(\mathbf{r})=\int \frac{P\left(\mathbf{r r} \mathbf{r}^{\prime}\right)\left(\mathbf{r}-\mathbf{r}^{\prime}\right)}{\left|\mathbf{r}-\mathbf{r}^{\prime}\right|^{3}} d \mathbf{r}^{\prime},
$$


with $P\left(\mathbf{r r}^{\prime}\right)$ the expectation

$$
P\left(\mathbf{r r}^{\prime}\right)=\left\langle\Psi(\mathbf{X})\left|\hat{P}\left(\mathbf{r r}^{\prime}\right)\right| \Psi(\mathbf{X})\right\rangle,
$$

of the pair operator

$$
\hat{P}\left(\mathbf{r r}^{\prime}\right)=\sum_{i, j}^{\prime} \delta\left(\mathbf{r}_{i}-\mathbf{r}\right) \delta\left(\mathbf{r}_{j}-\mathbf{r}^{\prime}\right) .
$$

The kinetic "force" $\mathbf{z}(\mathbf{r})$, representative of kinetic effects, is obtained from its quantal source, the single-particle density matrix $\gamma\left(\mathbf{r r}^{\prime}\right)$ :

$$
z_{\alpha}(\mathbf{r})=2 \sum_{\beta} \nabla_{\beta} t_{\alpha \beta}(\mathbf{r}),
$$

where the kinetic energy tensor

$$
t_{\alpha \beta}(\mathbf{r})=\left.\frac{1}{4}\left[\frac{\partial^{2}}{\partial r_{\alpha}^{\prime} \partial r_{\beta}^{\prime \prime}}+\frac{\partial^{2}}{\partial r_{\beta}^{\prime} \partial r_{\alpha}^{\prime \prime}}\right] \gamma\left(\mathbf{r}^{\prime} \mathbf{r}^{\prime \prime}\right)\right|_{\mathbf{r}^{\prime}=\mathbf{r}^{\prime \prime}=r},
$$

with $\gamma\left(\mathbf{r r}^{\prime}\right)$ the expectation

$$
\gamma\left(\mathbf{r r}^{\prime}\right)=\left\langle\Psi(\mathbf{X})\left|\hat{\gamma}\left(\mathbf{r r}{ }^{\prime}\right)\right| \Psi(\mathbf{X})\right\rangle
$$

of the density matrix operator

$$
\begin{gathered}
\hat{\gamma}\left(\mathbf{r r}^{\prime}\right)=\hat{A}+i \hat{B} \\
\hat{A}=\frac{1}{2} \sum_{j}\left[\delta\left(\mathbf{r}_{j}-\mathbf{r}\right) T_{j}(\mathbf{a})+\delta\left(\mathbf{r}_{j}-\mathbf{r}^{\prime}\right) T_{j}(-\mathbf{a})\right] \\
\hat{B}=-\frac{i}{2} \sum_{j}\left[\delta\left(\mathbf{r}_{j}-\mathbf{r}\right) T_{j}(\mathbf{a})-\delta\left(\mathbf{r}_{j}-\mathbf{r}^{\prime}\right) T_{j}(-\mathbf{a})\right],
\end{gathered}
$$

with $T_{j}(\mathbf{a})$ a translation operator such that $T_{j}(\mathbf{a}) \psi\left(\ldots \mathbf{r}_{j} \ldots\right)=\psi\left(\ldots \mathbf{r}_{j}+\mathbf{a}, \ldots\right)$. The differential density "force" $\mathbf{d}(\mathbf{r})$, representative of the density, is

$$
\mathbf{d}(\mathbf{r})=-\frac{1}{4} \nabla \nabla^{2} \rho(\mathbf{r}),
$$

the quantal source being the density $\rho(\mathbf{r})$. Finally, the internal magnetic "force" $\mathbf{i}(\mathbf{r})$ whose quantal source is the current density $\mathbf{j}(\mathbf{r})$ :

$$
i_{\alpha}(\mathbf{r})=\sum_{\beta} \nabla_{\beta} I_{\alpha \beta}(\mathbf{r}),
$$

with the tensor

$$
I_{\alpha \beta}(\mathbf{r})=\left[j_{\alpha}(\mathbf{r}) A_{\beta}(\mathbf{r})+j_{\beta}(\mathbf{r}) A_{\alpha}(\mathbf{r})\right]-\rho(\mathbf{r}) A_{\alpha}(\mathbf{r}) A_{\beta}(\mathbf{r}) .
$$

The components of the total energy - the kinetic $T$, electron-interaction $E_{\mathrm{ee}}$, internal magnetic $I$, and external $E_{\mathrm{ext}}$-can each be expressed in integral virial form in terms of the respective fields [2,27]:

$$
\begin{gathered}
T=-\frac{1}{2} \int \rho(\mathbf{r}) \mathbf{r} \cdot \mathcal{Z}(\mathbf{r}) d \mathbf{r}, \\
E_{\mathrm{ee}}=\int \rho(\mathbf{r}) \mathbf{r} \cdot \mathcal{E}_{\mathrm{ee}}(\mathbf{r}) d \mathbf{r}, \\
I=\int \rho(\mathbf{r}) \mathbf{r} \cdot \mathcal{I}(\mathbf{r}) d \mathbf{r}, \\
E_{\mathrm{ext}}=\int \rho(\mathbf{r}) \mathbf{r} \cdot \mathcal{F}^{\mathrm{ext}}(\mathbf{r}) d \mathbf{r} .
\end{gathered}
$$




\section{New Perspectives}

We next discuss the new understandings achieved via the single-electron perspective. They are valid for both ground and excited states.

(i) In addition to the external electrostatic $\mathcal{E}(\mathbf{r})$ and Lorentz $\mathcal{L}(\mathbf{r})$ fields, each electron experiences an internal field $\mathcal{F}^{\text {int }}(\mathbf{r})$. This field via its $\mathcal{E}_{\text {ee }}(\mathbf{r})$ component is representative not only of Coulomb correlations as one might expect, but also those due to the Pauli exclusion principle due to the antisymmetric nature of the wave function. Additionally, there is a component $\mathcal{Z}(\mathbf{r})$ representative of the motion of the electrons; a component $\mathcal{D}(\mathbf{r})$ representing the density, a fundamental property of the system [29]; and a term $\mathcal{I}(\mathbf{r})$ that arises as a consequence of the external magnetic field [27]. Hence, each electron experiences an internal field that encapsulates all the basic properties of the system. As in classical physics, in summing over all the electrons, the contribution of the internal field vanishes, leading thereby to Ehrenfest's (first law) theorem: $\int \rho(\mathbf{r}) \mathcal{F}^{\operatorname{ext}}(\mathbf{r}) d \mathbf{r}=0$. (In fact, each component of the internal field is shown to separately vanish.).

(ii) The "Quantal Newtonian" first law Equation (4) affords a rigorous physical interpretation of the external electrostatic potential $v(\mathbf{r})$ : it is the work done to move an electron from some reference point at infinity to its position at $\mathbf{r}$ in the force of a conservative field $\mathcal{F}(\mathbf{r})$ :

$$
v(\mathbf{r})=\int_{\infty}^{\mathbf{r}} \mathcal{F}\left(\mathbf{r}^{\prime}\right) \cdot d \ell^{\prime}
$$

where $\mathcal{F}(\mathbf{r})=\mathcal{F}^{\text {int }}(\mathbf{r})-\mathcal{L}(\mathbf{r})=\mathcal{E}_{\text {ee }}(\mathbf{r})-\mathcal{Z}(\mathbf{r})-\mathcal{D}(\mathbf{r})-\mathcal{I}(\mathbf{r})-\mathcal{L}(\mathbf{r})$. Since $\nabla \times \mathcal{F}(\mathbf{r})=0$, this work done is path-independent. Thus, we now understand, in the rigorous classical sense of a potential being the work done in a conservative field, that $v(\mathbf{r})$ represents a potential energy viz. that of an electron. Furthermore, that "classical" field is now explicitly defined. We reiterate that Equation (30) (or the "Quantal Newtonian" first law) is valid for arbitrary state. In other words, irrespective of whether the state is a ground, excited, or a degenerate state, the work done in the corresponding field $\mathcal{F}(\mathbf{r})$ is always the same, viz. $v(\mathbf{r})$.

(iii) What the physical interpretation of the potential $v(\mathbf{r})$ further shows is that it can no longer be thought of as an independent entity. It is intrinsically dependent upon all the properties of the system via the various components of the internal field $\mathcal{F}^{\text {int }}(\mathbf{r})$, and the Lorentz $\mathcal{L}(\mathbf{r})$ field through the current density $\mathbf{j}(\mathbf{r})$. Hence, the potential energy function $v(\mathbf{r})$ is comprised of the sum of constituent functions each representative of a property of the system.

(iv) As each component of the internal field $\mathcal{F}^{\text {int }}(\mathbf{r})$ (and the Lorentz field $\mathcal{L}(\mathbf{r})$ ) are obtained from quantal sources that are expectations of Hermitian operators taken with respect to the wave function $\Psi(\mathbf{X})$, we see that the field $\mathcal{F}(\mathbf{r})$ is a functional of $\Psi(\mathbf{X})$, i.e., $\mathcal{F}(\mathbf{r})=\mathcal{F}[\Psi(\mathbf{X})]$. Thus, (from Equation (30)), $v(\mathbf{r})$ is a functional of $\Psi(\mathbf{X}): v(\mathbf{r})=v[\Psi(\mathbf{X})]$. The functional $v[\Psi(\mathbf{X})]$ is exactly known [via Equation (30)].

(v) On substituting the functional $v[\Psi(\mathbf{X})]$ into Equation (1), the Schrödinger equation may then be written as

$$
\left[\frac{1}{2} \sum_{i}\left(\hat{\mathbf{p}}_{i}+\mathbf{A}\left(\mathbf{r}_{i}\right)\right)^{2}+\frac{1}{2} \sum_{i, j}^{\prime} \frac{1}{\left|\mathbf{r}_{i}-\mathbf{r}_{j}\right|}+\sum_{i} v[\Psi]\left(\mathbf{r}_{i}\right)\right] \Psi(\mathbf{X})=E \Psi(\mathbf{X}),
$$

or, equivalently, as

$$
\left[\frac{1}{2} \sum_{i}\left(\hat{\mathbf{p}}_{i}+\mathbf{A}\left(\mathbf{r}_{i}\right)\right)^{2}+\frac{1}{2} \sum_{i, j}^{\prime} \frac{1}{\left|\mathbf{r}_{i}-\mathbf{r}_{j}\right|}+\sum_{i} \int_{\infty}^{\mathbf{r}_{i}} \mathcal{F}[\Psi](\mathbf{r}) \cdot d \boldsymbol{\ell}\right] \Psi(\mathbf{X})=E \Psi(\mathbf{X}) .
$$

In general, with the Hamiltonian a functional of $\Psi(\mathbf{X})$, the Schrödinger equation can be written as $\hat{H}[\Psi(\mathbf{X})] \Psi(\mathbf{X})=E \Psi(\mathbf{X})$. In this manner, the intrinsic self-consistent nature of the Schrödinger equation becomes evident. (Recall that what is meant by the functional $v[\Psi]$ is that for each different $\Psi(\mathbf{X})$, one obtains a different $v[\Psi](\mathbf{r})$ ). To solve the equation (see Figure 1), one begins with an approximation to 
$\Psi(\mathbf{X})$. With this approximate $\Psi(\mathbf{X})$, one determines the various quantal sources and the fields $\mathcal{F}^{\text {int }}(\mathbf{r})$ and $\mathcal{L}(\mathbf{r})$ (for an external $\mathcal{B}(\mathbf{r})$ ), and the work done $v(\mathbf{r})$ in the sum of these fields. One then solves the integro-differential equation to determine a new approximate solution $\Psi(\mathbf{X})$ and eigenenergy $E$. This $\Psi(\mathbf{X})$, in turn, will lead to a new $v(\mathbf{r})$ (via Equation (30)), and by solution of the equation to a new $\Psi(\mathbf{X})$ and $E$. The process is continued until the input $\Psi(\mathbf{X})$ to determine $v[\Psi](\mathbf{r})$ leads to the same output $\Psi(\mathbf{X})$ on solution of the equation or, equivalently, until self-consistency is achieved. The exact $\hat{H}[\Psi], \Psi(\mathbf{X}), E$ are obtained in the final iteration of the self-consistency procedure.

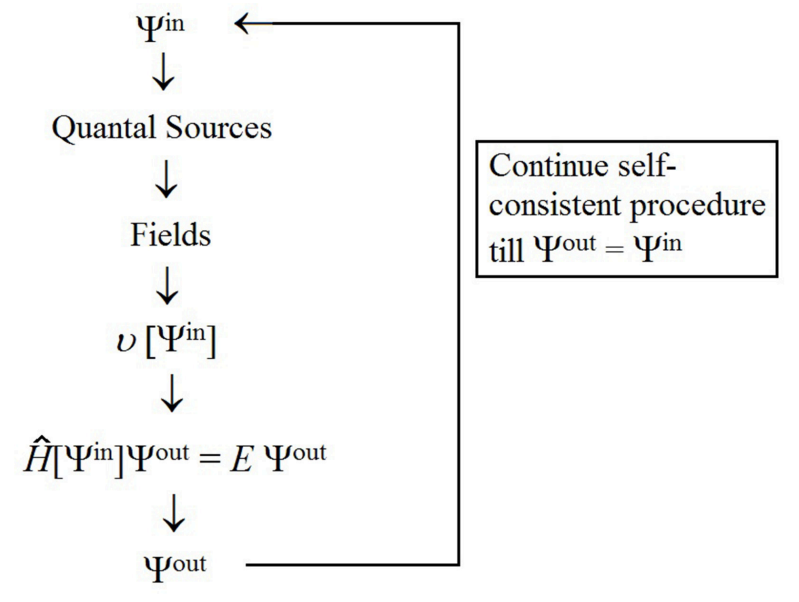

Figure 1. Self-consistent procedure for the solution of the Schrödinger equation.

In any self-consistent procedure, different external potentials $v(\mathbf{r})$ can be obtained based on the choice of the initial approximate input wave function $\Psi(\mathbf{X})$. In atoms, molecules or solids, the potential $v(\mathbf{r})$ obtained self-consistently would be Coulombic. In quantum dots, it would be harmonic, and so on. This is irrespective of the state of the system. One must begin with an educated accurate guess apropos to the physical system of interest for the initial input. Otherwise, one may not achieve self-consistency. Thus, for example, in self-consistent quantal density functional theory calculations on atoms [3,20,21], the initial input wave function for an atom is the solution of the prior atom of the Periodic Table. In general, for any self-consistent calculation, it is only after self-consistency is achieved that one must judge and test whether the solution is physically meaningful. (Note that in this manner, the external potential $v(\mathbf{r})$ and hence the Hamiltonian is determined self-consistently.).

In principle, the above procedure is mathematically entirely akin to the fully-self-consistent solution of the integro-differential equations of Hartree [14] and Hartree-Fock [15] theories, the Optimized Potential method [18,19], Quantal density functional theory [3], etc. In each of these cases, the corresponding integro-differential equations are of the form $\hat{H}\left[\zeta_{i}\right] \zeta_{i}(\mathbf{x})=\lambda_{i} \zeta_{i}(\mathbf{x})$, where $\hat{H}$ is the corresponding Hamiltonian and $\zeta_{i}, \lambda_{i}$ the single particle orbitals and eigenvalues, respectively. The Schrödinger equation written in self-consistent form- $\hat{H}[\Psi(\mathbf{X})] \Psi(\mathbf{X})=E \Psi(\mathbf{X})$-is of the same form but with the generalization to the many-electron system wave function $\Psi(\mathbf{X})$ and the corresponding eigenvalue $E$. Thus, we now understand that the Schrödinger equation too can be thought of as being a self-consistent equation. This perspective of Schrödinger theory is new.

We note that there exists a "Quantal Newtonian" first law for Hartree, Hartree-Fock, and local effective potential theories [2,3]. Hence, the external potential $v(\mathbf{r})$ of these theories can also be expressed as the work done in a conservative field, and thus replaced in the corresponding integro-differential equations by a known functional of the requisite Slater determinant. These theories are thereby further generalized.

(vi) Observe that in writing the Schrödinger equation as in Equations (31) and (32), the magnetic field $\mathcal{B}(\mathbf{r})$ now appears in the Hamiltonian explicitly via the Lorentz field $\mathcal{L}(\mathbf{r})$ (see Equation (30)). It is the intrinsic self-consistent nature of the equation that demands the presence of $\mathcal{B}(\mathbf{r})$ in the 
Hamiltonian. In other words, since the Hamiltonian $\hat{H}[\Psi]$ is being determined self-consistently, all the information of the physical system-electrons and fields-must be incorporated in it. (Of course, equivalently, the field $\mathcal{B}(\mathbf{r})$ could be expressed in terms of the vector potential $\mathbf{A}(\mathbf{r})$. This then shows that when written in self-consistent form, there exists another component of the Hamiltonian involving the vector potential.)

(vii) The presence of a solely electrostatic external field $\mathcal{E}(\mathbf{r})=-\nabla v(\mathbf{r})$ is a special case of the stationary state theory discussed above. This case then constitutes the description of matter as defined in the Introduction.

\section{Examples: Quantum Dots in a Ground and Excited State}

To explicate the new physics of stationary-state Schrödinger theory, we consider two different two-electron quantum dots or two-dimensional "artificial atoms" [30] in an external magnetostatic field [31,32]. The first quantum dot is in a ground state while the second is in a singlet excited state. The external scalar potential in the Hamiltonian of Equation (1) is then of the form $v(\mathbf{r})=\frac{1}{2} \omega_{0}^{2} r^{2}$. However, due to the presence of the magnetic field, (and in the symmetric gauge $\mathbf{A}(\mathbf{r})=\frac{1}{2} \mathcal{B}(\mathbf{r}) \times \mathbf{r}$ ), the electrons experience an effective harmonic force constant $k_{\text {eff }}=\omega_{0}^{2}+\omega_{L}^{2}$, with $\omega_{L}=B / 2$ the Larmor frequency. Following Taut [31], the wave function for the quantum dot in its ground state is derived [27] to be

$$
\Psi_{0}\left(\mathbf{r}_{1} \mathbf{r}_{2}\right)=C_{0} e^{-\Omega\left(R^{2}+\frac{1}{4} r^{2}\right)}(1+r),
$$

where $\mathbf{R}=\left(\mathbf{r}_{1}+\mathbf{r}_{2}\right) / 2, r=\left|\mathbf{r}_{1}-\mathbf{r}_{2}\right|, C_{0}=\Omega^{\frac{3}{2}} / \pi[2+\Omega+\sqrt{2 \pi \Omega}]^{\frac{1}{2}}, \Omega=\sqrt{k_{\text {eff }}}, k_{\text {eff }}=1$, with energy 3.000000 a.u. The wave function for the dot in the singlet state is derived [33] as

$$
\begin{aligned}
\Psi_{1}\left(\mathbf{r}_{1} \mathbf{r}_{2}\right)=C_{1} e^{-\Omega\left(R^{2}+\frac{1}{4} r^{2}\right)}[1 & +r+\left(\frac{\Omega}{4}-0.436815\right) r^{2} \\
& \left.+\left(\frac{\Omega}{4}-0.353786\right) r^{3}\right]
\end{aligned}
$$

where $C_{1}=0.108563, \Omega=\sqrt{k_{\text {eff }}}, k_{\text {eff }}=0.471716$, with energy $E_{1}=3.434076$ a.u. (The purpose of employing two different quantum dots is because for the two different force constants $k_{\text {eff }}$, the corresponding wave functions, and hence the majority of the properties, can be obtained in closed analytical form.).

In Figures 2 and 3, we plot the corresponding electron-interaction $\mathcal{E}_{\text {ee }}(\mathbf{r})$, kinetic $\mathcal{Z}(\mathbf{r})$, and differential density $\mathcal{D}(\mathbf{r})$ components of the internal $\mathcal{F}^{\text {int }}(\mathbf{r})$ field. Observe that $-\mathcal{E}_{\text {ee }}(\mathbf{r})+\mathcal{Z}(\mathbf{r})+$ $\mathcal{D}(\mathbf{r})=-k_{\text {eff }} r$. This then demonstrates the satisfaction of the "Quantal Newtonian" first law of Equation (4) (for the details of these calculations, see [27,34]).

The examples of the quantum dots above can be thought of as being the final iteration of the self-consistent procedure in which the exact potential $v(\mathbf{r})$, wave function $\Psi\left(\mathbf{r}_{1} \mathbf{r}_{2}\right)$, and energy $E$ are obtained. To see this, consider the initial choice of solutions to be the following:

$$
\psi_{0}\left(\mathbf{r}_{1} \mathbf{r}_{2}\right)=C_{0} e^{-\Omega_{0}\left(R^{2}+\frac{1}{4} r^{2}\right)}\left(1+a_{0} r\right),
$$

and

$$
\psi_{1}\left(\mathbf{r}_{1} \mathbf{r}_{2}\right)=C_{1} e^{-\Omega_{1}\left(R^{2}+\frac{1}{4} r^{2}\right)}\left(1+a_{1} r+b_{1} r^{2}+c_{1} r^{3}\right),
$$

where $C_{0}, C_{1}, \Omega_{0}, \Omega_{1}, a_{0}, a_{1}, b_{1}, c_{1}$ are constants. Let us next assume that, for some random iteration, the values of these coefficients turn out to be $C_{0}=0.135646, \Omega_{0}=1.000000, a_{0}=1.000000$; $C_{1}=0.108563, \Omega_{1}=0.686816, a_{1}=1.000000, b_{1}=-0.265111, c_{1}=-0.182082$. One then determines the various fields from the corresponding solutions and plots them. On adding the fields $\mathcal{D}(\mathbf{r})$ and $\mathcal{Z}(\mathbf{r})$, one obtains the dot-dash lines as shown in Figures 2 and 3 for $(\mathcal{D}(\mathbf{r})+\mathcal{Z}(\mathbf{r}))$. Adding $-\mathcal{E}_{\text {ee }}(\mathbf{r})$ to these lines, one then obtains a straight (dashed) line $-k_{\text {eff }} r$ in each case, i.e., the gradient of the corresponding effective scalar potential. On substituting this $\frac{1}{2} k_{\text {eff }} r^{2}$ back into the Schrödinger equation 
and solving, one obtains the same wave functions $\Psi_{0}, \Psi_{1}$ and energies $E_{0}, E_{1}$ as that of Equations (33) and (34). Additionally, it becomes clear that the potential $v(\mathbf{r})$ is harmonic. This then constitutes the final iteration of the self-consistency procedure and shows the intrinsic self-consistent nature of the Schrödinger equation.

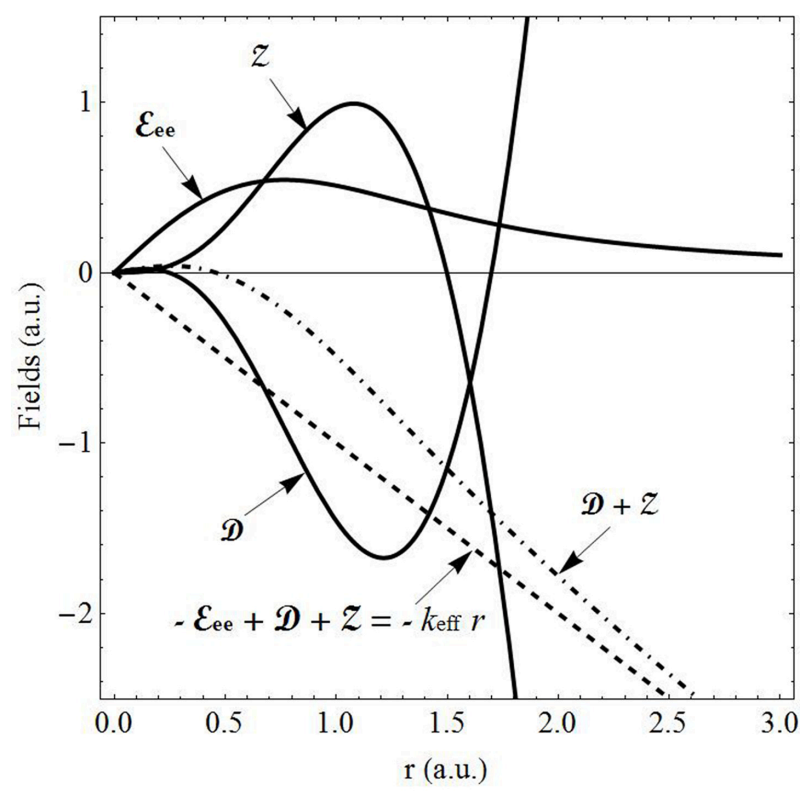

Figure 2. The electron-interaction $\mathcal{E}_{\text {ee }}(\mathbf{r})$, kinetic $\mathcal{Z}(\mathbf{r})$, and differential density $\mathcal{D}(\mathbf{r})$ components of the internal field $\mathcal{F}^{\text {int }}(\mathbf{r})$ for a quantum dot in a magnetic field in its ground state. The sums $\mathcal{D}(\mathbf{r})+\mathcal{Z}(\mathbf{r})$, and $-\mathcal{E}_{\text {ee }}(\mathbf{r})+\mathcal{Z}(\mathbf{r})+\mathcal{D}(\mathbf{r})=-k_{\text {eff }} r$ with $k_{\text {eff }}=1$ are also plotted.

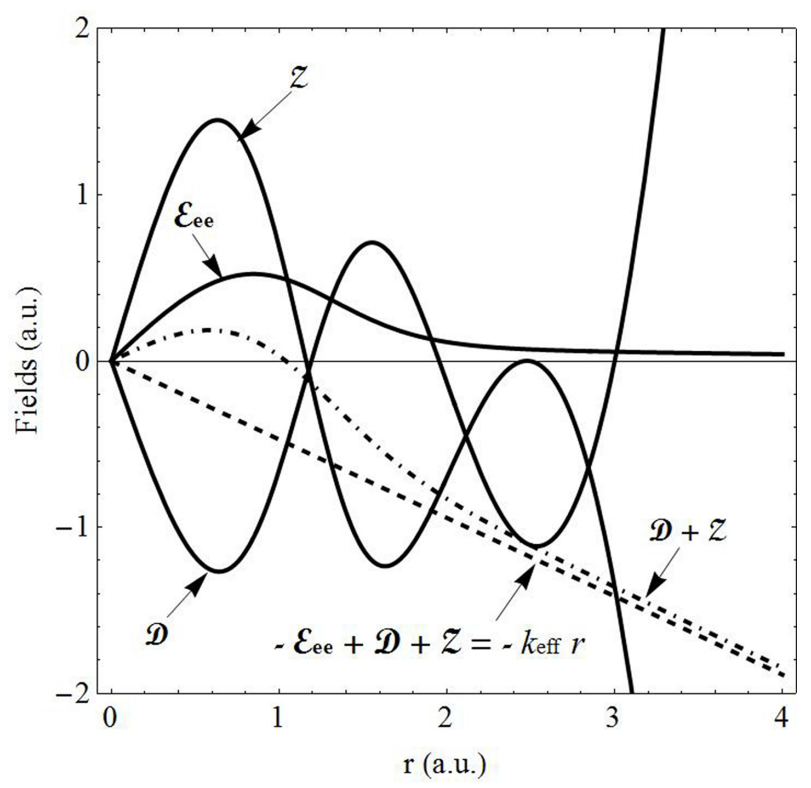

Figure 3. Same as in Figure 2 but for a quantum dot in a magnetic field in its first excited singlet state with $k_{\text {eff }}=0.471716$. 


\section{Time-Dependent Theory: “Quantal Newtonian" Second Law}

The above conclusions are generalizable to the time-dependent Schrödinger equation. For simplicity, let us first consider the external field to be $\mathcal{F}^{\text {ext }}(\mathbf{r} t)=\mathcal{E}(\mathbf{r} t)=-\nabla v(\mathbf{r} t)$. In this case, the "Quantal Newtonian" second law for each electron-the quantal equivalent to Newton's second law of Equation (2)—is $[2,4,5]$.

$$
\mathcal{F}^{\text {ext }}(\mathbf{r} t)+\mathcal{F}^{\text {int }}(\mathbf{r} t)=\mathcal{J}(\mathbf{r} t)
$$

where $\mathcal{F}^{\text {int }}(\mathbf{r} t)$ is given by the TD version of Equation (12) (without the $\mathcal{I}(\mathbf{r} t)$ term) and the response of the electron is described by the current density field $\mathcal{J}(\mathbf{r} t)=(1 / \rho(\mathbf{r} t)) \partial \mathbf{j}(\mathbf{r} t) / \partial t$. The corresponding external potential energy $v[\Psi](\mathbf{r} t)$ functional of the wave function $\Psi(\mathbf{X} t)$ as obtained from Equation (37) is then the work done at each instant of time in a conservative field:

$$
v[\Psi](\mathbf{r} t)=\int_{\infty}^{\mathbf{r}} \mathcal{F}\left(\mathbf{r}^{\prime} t\right) \cdot d \ell^{\prime},
$$

where $\mathcal{F}(\mathbf{r} t)=\mathcal{F}^{\text {int }}(\mathbf{r} t)-\mathcal{J}(\mathbf{r} t)$, with the TD self-consistent Schrödinger equation being

$$
\left[\frac{1}{2} \sum_{i} \hat{\mathbf{p}}_{i}^{2}+\frac{1}{2} \sum_{i, j}^{\prime} \frac{1}{\left|\mathbf{r}_{i}-\mathbf{r}_{j}\right|}+\sum_{i} v[\Psi]\left(\mathbf{r}_{i} t\right)\right] \Psi(\mathbf{X} t)=i \frac{\partial \Psi(\mathbf{X} t)}{\partial t} .
$$

Since $\boldsymbol{\nabla} \times \mathcal{F}(\mathbf{r} t)=0$, the work done $v(\mathbf{r} t)$ at each instant of time is path-independent and thus a potential energy. Again, on summing Equation (37) over all the electrons, the contribution of $\mathcal{F}^{\text {int }}(\mathbf{r} t)$ vanishes, leading to Ehrenfest's (second law) theorem $\int \rho(\mathbf{r} t)\left[\mathcal{F}^{\text {ext }}(\mathbf{r} t)-\mathcal{J}(\mathbf{r} t)\right] d \mathbf{r}=0$. The further generalization of the "Quantal Newtonian" second law to the case of an external TD electromagnetic field with $\mathcal{E}(\mathbf{r})=-\boldsymbol{\nabla} v(\mathbf{r}), \mathbf{E}(\mathbf{r} t)=-\boldsymbol{\nabla} \phi(\mathbf{r} t)-\partial \mathbf{A}(\mathbf{r} t) / \partial t, \mathcal{B}(\mathbf{r} t)=\boldsymbol{\nabla} \times \mathbf{A}(\mathbf{r} t)$, is given in [7]. The resulting self-consistent time-dependent Schrödinger equation then follows.

As an example of the insights for the time-dependent case, consider the two two-electron quantum dots in an external magnetostatic field $\mathcal{B}(\mathbf{r})=\boldsymbol{\nabla} \times \mathbf{A}(\mathbf{r})$ perturbed by a time-dependent electric field $\mathbf{E}(t)$. The wave function of this system [35], known as the Generalized Kohn Theorem [36-41], is comprised of a phase factor times the unperturbed wave function in which the coordinates of each electron are translated by a time-dependent function that satisfies the classical equation of motion. Hence, if the unperturbed wave function is known, the time evolution of all properties is known. As the wave functions for the unperturbed quantum dots in their ground and excited states are given by Equations (33) and (34), the corresponding solutions of the time-dependent Schrödinger equation, and therefore of all the various fields, is obtained. At the initial time, $t=0$, the results are those of Figures 2 and 3 . The evolution of observables that are expectations of non-differential operators such as the density $\rho(\mathbf{r} t)$, the electron-interaction field $\mathcal{E}_{\text {ee }}(\mathbf{r} t)$, etc., are simply the time-independent functions shifted in time.

\section{Conclusions}

In conclusion, we have provided a new perspective on the Schrödinger theory of electrons in electromagnetic fields. This perspective, together with new insights, is arrived at via the "Quantal Newtonian" first and second laws for each electron. These laws are valid, respectively, for stationary-state and time-dependent Schrödinger theory. A principal understanding is that the scalar potential energy of an electron $\{v(\mathbf{r}) / v(\mathbf{r} t)\}$ is a known functional of the wave function $\{\Psi(\mathbf{X}) / \Psi(\mathbf{X} t)\}$. As such, the Hamiltonian $\{\hat{H} / \hat{H}(t)\}$ is a functional of the wave function: $\{\hat{H}[\Psi(\mathbf{X})] / \hat{H}[\Psi(\mathbf{X} t)]\}$. Hence, the time-independent Schrödinger equation can be written as $\hat{H}[\Psi(\mathbf{X})] \Psi(\mathbf{X})=E \Psi(\mathbf{X})$ and the time-dependent equation as $\hat{H}[\Psi(\mathbf{X} t)] \Psi(\mathbf{X} t)=i \partial \Psi(\mathbf{X} t) / \partial t$. Thus, the Schrödinger equation can now be thought of as one whose solution can be obtained self-consistently. The concept of the Schrödinger equation as being a self-consistent one is new. A path for the determination of the exact wave function is thus formulated. Such a path is feasible 
given the advent of present-day high computing power. The self-consistent nature of the Schrödinger equation is, however, demonstrated for the examples of a two-electron quantum dot in a magnetic field, one in a ground state and the other in an excited state. What this perspective on Schrödinger theory also shows is that when the scalar potential $\{v(\mathbf{r}) / v(\mathbf{r} t)\}$ is a known function, then the corresponding Schrödinger equations $\hat{H} \Psi(\mathbf{X})=E \Psi(\mathbf{X})$ and $\hat{H}(t) \Psi(\mathbf{X} t)=i \partial \Psi(\mathbf{X} t) / \partial t$ constitute a special case of the self-consistent form.

A second understanding achieved is that it is now possible to write the scalar potential $\{v(\mathbf{r}) / v(\mathbf{r} t)\}$ as the sum of component functions each of which is representative of a specific property of the system such as the correlations due to the Pauli exclusion principle and Coulomb repulsion, kinetic and magnetic effects, and the electron density. Although the component functions will differ depending upon the state, the scalar potential $\{v(\mathbf{r}) / v(\mathbf{r} t)\}$ remains the same. Such a property-related division of the scalar potential is also shown by the two examples of the quantum dots in a magnetostatic field given in the text.

Finally, an interesting observation is that, in its self-consistent form, in addition to the vector potential, which appears in the Schrödinger equation as a consequence of the correspondence principle, the magnetic field now appears too in the equation because of the "Quantal Newtonian" laws. Ex post facto, we now understand that this must be the case as the Hamiltonian itself is being determined self-consistently.

It is interesting to compare the self-consistent method for the determination of the wave function in the stationary ground state case to that of the variational method [23]. The latter is associated principally with the property of the total energy. An approximate parametrized variational wave function correct to $O(\delta)$ leads to an upper bound for the energy that is correct to $O\left(\delta^{2}\right)$. Such a wave function is accurate in the region where the principal contribution to the energy arises. However, all other observables obtained as the expectation of Hermitian single- and two-particle operators are correct only to the same order as that of the wave function, viz. to $O(\delta)$. A better approximate variational wave function is one that leads to a lower value of the energy. There is no guarantee that other observables representative of different regions of configuration space are thereby more accurate. On the other hand, in the self-consistent procedure, achieved say to a desired accuracy of five decimal places, all the properties are correct to the same degree of accuracy. An improved wave function would be one correct to a greater decimal accuracy. As a point of note, the constrained-search variational method [24-26] expands the variational space of approximate parametrized wave functions by considering the wave function $\Psi$ to be a functional of a function $\chi$, i.e., $\Psi=\Psi[\chi]$. One searches over all functions $\chi$ such that the wave function $\Psi[\chi]$ is normalized, gives the exact (theoretical or experimental) value of an observable, while leading to a rigorous upper bound to the energy. In this manner, the wave function functional $\Psi[\chi]$ is accurate not only in the region contributing to the energy, but also that of the observable. The wave function $\Psi[\chi]$ is, however, still approximate. The self-consistent solution of the Schrödinger equation, on the other hand, is exact to the degree required, throughout configuration space.

We do not address here the broader numerical procedural aspects of the self-consistency, nor the implications of the explicit presence of the magnetic field in it. These issues constitute current and future research.

Acknowledgments: The authors acknowledge Lou Massa and Marlina Slamet for their critique of the paper. V.S. is supported in part by the Research Foundation of the City University of New York. X.-Y.P. is supported by the National Natural Science Foundation of China (Grant No. 11275100).

Author Contributions: The paper is a result of years of collaboration between the authors. V.S. wrote the paper.

Conflicts of Interest: The authors declare no conflict of interest.

\section{References and Notes}

1. Sahni, V.; Pan, X.-Y. Study of the Schrödinger Theory for Electrons in External Fields. Available online: http:/ / meetings.aps.org/link/BAPS.2016.MAR.K31.13 (accessed on 9 March 2017). 
2. Sahni, V. Quantal Density Functional Theory, 2nd ed.; Springer: Berlin/Heidelberg, Germany, 2016.

3. Sahni, V. Quantal Density Functional Theory II: Approximation Methods and Applications; Springer: Berlin/Heidelberg, Germany, 2010.

4. Qian. Z.; Sahni, V. Quantum mechanical interpretation of the time-dependent density functional theory. Phys. Lett. A 1998, 247, 303-308.

5. Qian. Z.; Sahni, V. Time-dependent differential virial theorems. Int. J. Quantum Chem. 2000, 78, 341-347.

6. Qian. Z.; Sahni, V. Sum rules and properties in time-dependent density functional theory. Phys. Rev. A 2001, 63, 042508 .

7. Sahni, V.; Pan, X.-Y.; Yang, T. Electron correlations in local effective potential theory. Computation 2016, 4, 30.

8. Sahni, V. Quantum-mechanical interpretation of density functional theory. In Density Functional Theory III; Nalewajski, R.F., Ed.; Springer: Berlin/Heidelberg, Germany, 1996; pp. 1-39.

9. Sahni, V. Physical interpretation of density functional theory and of its representation of the Hartree-Fock and Hartree theories. Phys. Rev. A 1997, 55, 1846.

10. Holas, A.; March, N.H. Exact exchange-correlation potential and approximate exchange potential in terms of density matrices. Phys. Rev. A 1995, 51, 2040-2048.

11. Harbola, M.K.; Sahni, V. Quantum-Mechanical Interpretation of the Exchange-Correlation Potential of Kohn-Sham Density-Functional Theory. Phys. Rev. Lett. 1989, 62, 489-492.

12. Sahni, V.; Harbola, M.K. Quantum-Mechanical Interpretation of the Local Many-Body Potential of Density-Functional Theory. Int. J. Quantum Chem. 1990, 24, 569-584.

13. Sahni, V.; Slamet, M. Interpretation of Electron Correlation in the Local Density Approximation for Exchange. Phys. Rev. B 1993, 48, 1910-1913.

14. Hartree, D.R. The Calculation of Atomic Structures; Wiley: New York, NY, USA, 1957.

15. Fischer, C.F. The Hartree-Fock Theory for Atoms; Wiley: New York, NY, USA, 1977.

16. Hohenberg, P.; Kohn, W. Inhomogeneous electron gas. Phys. Rev. 1964, 136, B864.

17. Kohn. W.; Sham, L.J. Self-consistent equations including exchange and correlation effects. Phys. Rev. 1965, 140, A1133.

18. Talman, J.D.; Shadwick, W.F. Optimized effective atomic central potential. Phys. Rev. A 1976, 14, 36-40.

19. Engel, E.; Vosko, S.H. Accurate optimized-potential-model solutions for spherical spin-polarized atoms: Evidence for limitations of the exchange-only local spin-density and generalized-gradient approximations. Phys. Rev. A 1993, 47, 2800-2811.

20. Sahni, V.; Qian, Z.; Sen, K.D. Atomic Shell Structure in Hartree Theory. J. Chem. Phys. 2001, 114, $8784-8788$.

21. Sahni, V.; Li, Y.; Harbola, M.K. Atomic Structure in the Pauli-Correlated Approximation. Phys. Rev. A 1992, 45, 1434-1448.

22. Aharonov, Y.; Bohm, D. Significance of Electromagnetic Potentials in the Quantum Theory. Phys. Rev. 1959, 115, 485, doi:10.1103/PhysRev.115.485.

23. Moiseiwitsch, B.L. Variational Principles; Wiley: London, UK, 1966.

24. Pan, X.-Y.; Sahni, V.; Massa, L. Determination of a wave function functional. Phys. Rev. Lett. 2004, 93, 130401.

25. Pan, X.-Y.; Slamet, M.; Sahni, V. Wave function functionals. Phys. Rev. A 2010, 81, 042524.

26. Slamet, M.; Pan, X.-Y.; Sahni, V. Wave function functionals for the density. Phys. Rev. A 2011, 84, 052504.

27. Yang, T.; Pan, X.-Y.; Sahni, V. Quantal density functional theory in the presence of a magnetic field. Phys. Rev. A 2011, 83, 042518.

28. Holas, A.; March, N.H. Density matrices and density functionals in strong magnetic fields. Phys. Rev. A 1997, 56,4595 .

29. According to the first theorem of Hohenberg-Kohn [16], the nondegenerate ground state density $\rho(\mathbf{r})$ is a basic variable of quantum mechanics: A bijective relationship between this density and the external potential $v(\mathbf{r})$ is proved. Hence, knowledge of this $\rho(\mathbf{r})$ uniquely determines the potential $v(\mathbf{r})$ to within a constant, which in turn determines the Hamiltonian of the electrons, and via the Schrödinger equation, the wave functions of the system. Hence, although this density is a one-body property, it incorporates all the many-body information such that both ground and excited state properties of a system can be determined via its knowledge. We note that in the "Quantal Newtonian" first law, the density is not restricted to the nondegenerate ground state but rather is arbitrary in that it corresponds to the state being considered.

30. Ashoori, R.C. Electrons in artificial atoms. Nature 1996, 379, 413-419. 
31. Taut, M. Two electrons in a homogeneous magnetic field: Particular analytical solutions. J. Phys. A 1994, 27, 1045; Corrigenda. J. Phys. A 1994, 27, 4723.

32. Taut, M.; Eschrig, H. Exact solutions for a two-electron quantum dot model in a magnetic field and application to more complex systems. Z. Phys. Chem. 2010, 224, 631-649.

33. Slamet, M.; Sahni, V. Study of a Quantum Dot in an Excited State. Available online: http://meetings.aps. org/link/BAPS.2016.MAR.H31.10 (accessed on 9 March 2017).

34. Slamet, M.; Sahni, V. Electron correlations in an excited state of a Quantum Dot in a uniform magnetic field. Phys. Rev. A 2017, in preparation.

35. Zhu, H.M.; Chen, J.-W.; Pan, X.-Y.; Sahni, V. Wave function for harmonically confined electrons in time-dependent electric and magnetostatic fields. J. Chem. Phys. 2014, 140, 024318.

36. Kohn, W. Cyclotron resonance and de Haas-Van Alphen oscillations of an interacting electron gas. Phys. Rev. 1961, 123, 1242, doi:10.1103/PhysRev.123.1242.

37. Brey, L.; Johnson, N.F.; Halperin, B.I. Optical and magneto-optical absorption in parabolic quantum wells. Phys. Rev. B 1989, 40, 10647-10649, doi:10.1103/PhysRevB.40.10647.

38. Yip, S.K. Magneto-optical absorption by electrons in the presence of parabolic confinement potentials. Phys. Rev. B 1991, 43, 1707-1718, doi:10.1103/PhysRevB.43.1707.

39. Maksym, P.A.; Chakraborty, T. Quantum dots in a magnetic field: Role of electron-electron interactions. Phys. Rev. Lett. 1990, 65, 108-111, doi:doi.org/10.1103/PhysRevLett.65.108.

40. Peters, F.M. Magneto-optics in parabolic quantum dots. Phys. Rev. B 1990, 42, 1486-1487, doi:10.1103/PhysRevB.42.1486.

41. Dobson, J. Harmonic potential theorem: Implications for approximate many-body theories. Phys. Rev. Lett. 1994, 73, 2244-2247, doi:10.1103/PhysRevLett.73.2244.

(C) 2017 by the authors. Licensee MDPI, Basel, Switzerland. This article is an open access article distributed under the terms and conditions of the Creative Commons Attribution (CC BY) license (http:/ / creativecommons.org/licenses/by/4.0/). 